\title{
NUEVAS TECNOLOGÍAS EN ANÁLISIS DE INTELIGENCIA COMPETITIVA. CASOS PRÁCTICOS
}

\author{
Pilar Ramírez-Calvo, Ana-Cristina Triviño, Aurelio Berges-García, Juan- \\ Manuel Meneses-Chaus y José-Fernán Martínez
}
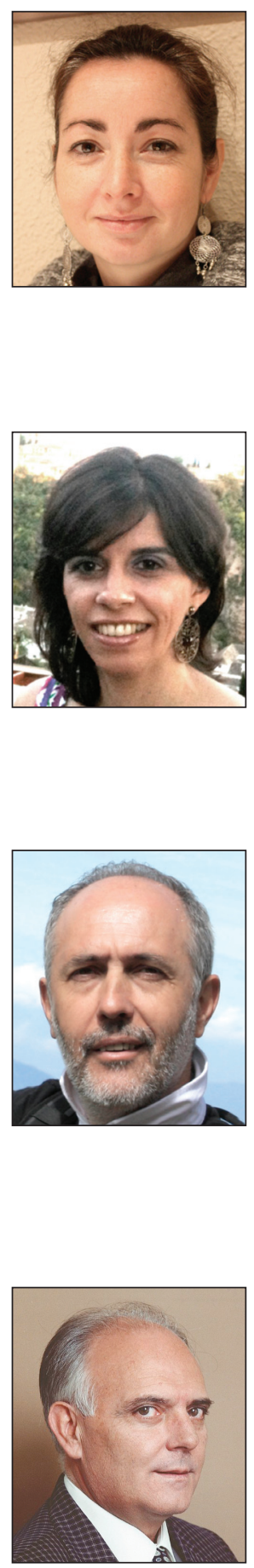

Pilar Ramírez-Calvo, doctora en ciencia y tecnología de alimentos y licenciada en bioquímica, es responsable del área de VT\&IC en e-Intelligent, gestionando diferentes proyectos en colaboración con organismos públicos y empresas privadas. Analista de inteligencia con más de 10 años de experiencia en entornos de I+D+i y más de 150 informes de estrategia empresarial como: patentes, estados del arte, nuevos mercados, socios, competidores, benchmarking, SWOTs, etc. Responsable de certificación 166.006:2011 de VT\&IC en e-Intelligent. Participante en congresos, seminarios y workshops internacionales, con publicaciones científicas y patentes.

http://orcid.org/0000-0002-4014-686X

e-Intelligent

Av. de la Albufera, 321, pl. 4, ofic. 10. 28031 Madrid, España pilar.ramirez@e-intelligent.es

Ana-Cristina Triviño es gerente y responsable de desarrollo de negocio del área de Vigilancia tecnológica e Inteligencia competitiva (VT\&IC): Vicubo Cloud. Consultora y formadora para diferentes unidades de VT\&IC de ámbito regional, nacional e internacional. Dirección de proyectos web de: marketing online, usabilidad, e implantación de soluciones de VT\&IC, buscadores, gestores documentales, gestores de contenidos, e-learning, businness intelligence y data mining. Participa activamente en jornadas, seminarios y máster. Postgrado Executive en inteligencia de negocio (BI) por la Escuela de Organización Industrial (EOI) y Licenciada en documentación por la Universidad Carlos III de Madrid. http://orcid.org/0000-0003-3387-5594

e-Intelligent

Av. de la Albufera, 321, pl. 4, of. 10. 28031 Madrid, España cristina.trivino@e-intelligent.es

Aurelio Berges-García es director del Grupo Gridat e investigador y profesor de telecomunicaciones en la Universidad Politécnica de Madrid. Investigador en TICs relacionadas con la recuperación de información inteligente, sistemas de búsqueda, minería de datos y sistemas para la VT\&IC. Asesor en el desarrollo e implantación de herramientas de VT\&IC, CRM y ERP y experto en sistemas de gestión del conocimiento, como el Sistema Madritd. Posee una larga experiencia como coordinador en más de 80 proyectos de I+D, nacionales e internacionales. Es autor de artículos de revista y ponencias de congreso, así como de varios libros.

http://orcid.org/0000-0003-0597-6806

Univ. Politécnica de Madrid, Escuela Univ. de Ingeniería Técnica de Telecomunicación Depto. de Ingeniería y Arquitecturas Telemáticas (Diatel) Carr. de Valencia, Km. 7. 28031 Madrid, España aurelio.berges@upm.es

Juan-Manuel Meneses-Chaus es catedrático del Área de Tecnología Electrónica de la Universidad Politécnica de Madrid (UPM) y director del Centro de Investigación en Tecnologías SW. Es experto en síntesis y diseño de arquitecturas digitales de alta velocidad y diseño de sistemas de inteligencia ambiental en aplicaciones de salud. Dirigió la OTRI de la UPM hasta 2008. Creador y responsable del círculo de innovación en TIC, dedicado a actividades VT\&IC. Colabora en programas de IP y es asesor de la $O M P I$. Ha dirigido tesis doctorales y participado en 77 proyectos. Es autor de más de 95 publicaciones en revistas y congresos.

http://orcid.org/0000-0002-0569-2910

Univ. Politécnica de Madrid, Escuela Univ. de Ingeniería Técnica de Telecomunicación Depto. de Tecnologías del Software y Sistemas multimedia para la Sostenibilidad (Citsem) Carr. de Valencia, Km. 7. 28031 Madrid, España juan.meneses@upm.es 


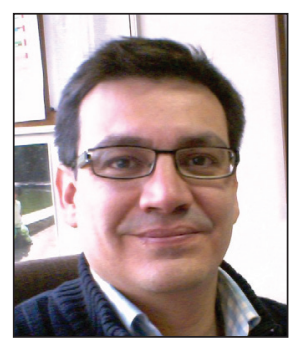

José-Fernán Martínez, de telecomunicación y profesor titular de la Universidad Politécnica de Madrid (UPM), es responsable de proyectos de investigación en IT. Sus áreas de interés son, entre otras, internet de las cosas, ciudades inteligentes, redes inalámbricas de sensores y actuadores (WSAN). Es autor de publicaciones nacionales e internacionales, revisor técnico de eventos internacionales sobre telemática y miembro de comités técnicos y científicos. Ha participado en varios proyectos internacionales y europeos con financiación pública, y también en contratos de investigación con empresas de IT. http://orcid.org/0000-0002-0569-2910

Univ. Politécnica de Madrid, Escuela Univ. de Ingeniería Técnica de Telecomunicación Depto. de Tecnologías del Software y Sistemas multimedia para la Sostenibilidad (Citsem) Carr. de Valencia, Km. 7. 28031 Madrid, España jf.martinez@upm.es

\section{Resumen}

Las nuevas tecnologías basadas en internet están transformando los modelos de negocio en todo el mundo. Se plantea el uso de las nuevas plataformas de vigilancia tecnológica e inteligencia competitiva en unos casos prácticos en el entorno empresarial. Se repasan las diferentes fuentes de información en combinación con otros datos (como información tecnológica y datos financieros) para la creación de inteligencia. Las herramientas de análisis permiten detectar relaciones entre los datos de manera que las organizaciones pueden identificar cambios, adaptar sus productos a nuevos consumidores o anticipar los movimientos del mercado. Se describen 3 casos prácticos usando la solución VicuboCloud en la recolección y análisis de información para la toma de decisiones.

\section{Palabras clave}

Vigilancia tecnológica, Inteligencia competitiva, Inteligencia empresarial, Cloud computing, Análisis de datos, Casos prácticos.

Title: New technologies for analysis of business intelligence. Practical cases

\section{Abstract}

New internet-based technologies are transforming the business model around the world. The use of new competitive watch and intelligence platforms is discussed. We review the information sources used in combination with other data (e.g., financial and technological data) to create intelligence. Analytical tools allow the detection of connections between data so that the company can identify changes, adapt products to new customers, or anticipate market movements. Three practical cases are presented using the VicuboCloud solution to collect and analyze information for decision-making.

\section{Keywords}

Technological watch, Competitive intelligence, Business intelligence, Cloud computing, Data analysis, Practical cases.

Ramírez-Calvo, Pilar; Triviño, Ana-Cristina; Berges-García, Aurelio; Meneses-Chaus, Juan-Manuel; Martínez, José-Fernán (2013). "Nuevas tecnologías en análisis de inteligencia competitiva. Casos prácticos". El profesional de la información, septiembre-octubre, v. 22, n. 5, pp. 448-454.

http://dx.doi.org/10.3145/epi.2013.sep.10

\section{Introducción}

La inteligencia competitiva (IC) ha crecido en las últimas décadas para convertirse en una actividad de gran importancia en las empresas (Fuld, 1985; McKinnon; Burns, 1992). Puede definirse como un proceso de creación de relaciones entre información de diferentes fuentes con implicaciones potenciales para la mejora de la gestión de negocio y el apoyo a la toma de decisiones estratégicas en las áreas de marketing, desarrollo de producto, mercado e I+D+i, entre otras. La IC va más allá de la investigación de mercado o la vigilancia tecnológica, centrándose en todos los aspectos del entorno de la empresa para producir inteligencia (conocimiento del entorno para saber cómo actuar de forma óptima) en aspectos clave como la detección temprana de amenazas y oportunidades, identificación de mercados y clientes, monitorización y evaluación de competidores y apoyo a la planificación estratégica.
La cantidad de información disponible generada cada día, la competencia a nivel global, el énfasis en la gestión de la calidad, y la necesidad de disminuir el riesgo en la toma de decisiones ha llevado a utilizar medios tecnológicos en la práctica de la IC. Además, la utilización conjunta de las redes sociales amplía su alcance, incluyendo información adicional táctica y estratégica que puede ser añadida al conocimiento de la compañía.

Tradicionalmente, el proceso de IC se compone de cuatro etapas (Herring, 1999; Fuld \& Company, 2002):

1. Planificación y dirección. Determinación de las necesidades de información estratégica o factores críticos de vigilancia (FCV): datos del entorno esenciales para el negocio.

2. Recolección y almacenamiento de datos. Buscar qué fuentes fiables y accesibles se pueden usar y recabar datos de ellas. Filtrar los resultados eligiendo los elementos de interés que se incluirán en la base de datos del proyecto. 


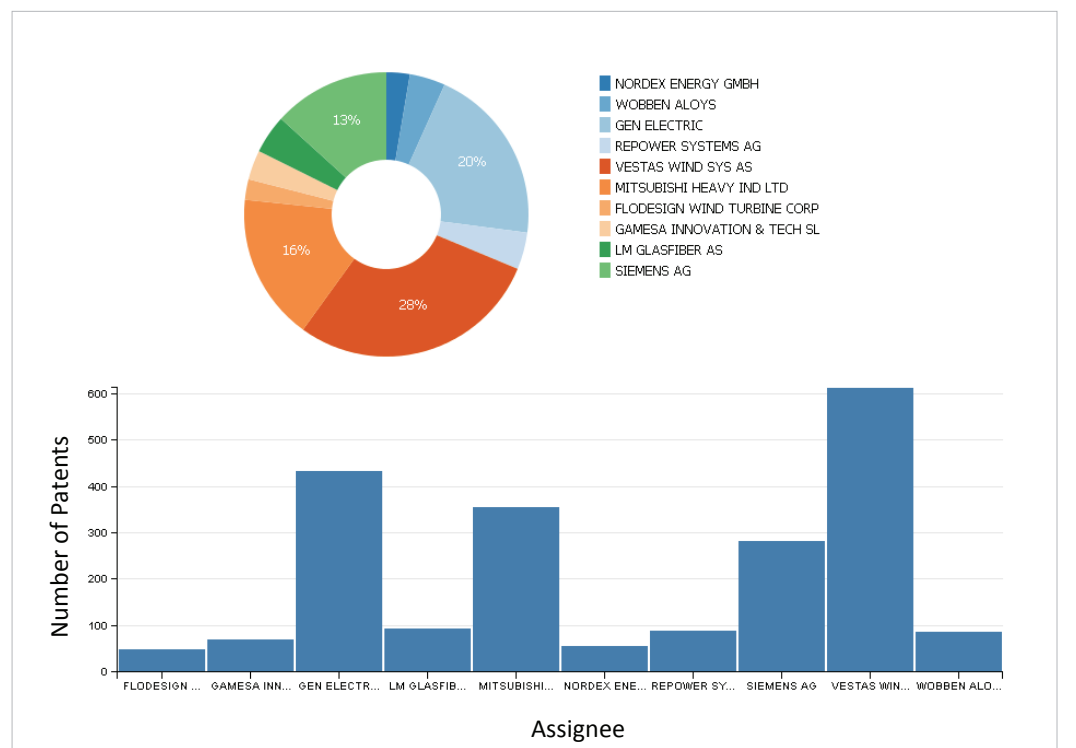

Figura 1. Top 10 líderes de patentes entre los suministradores de energía eólica (fuente: Espacenet)

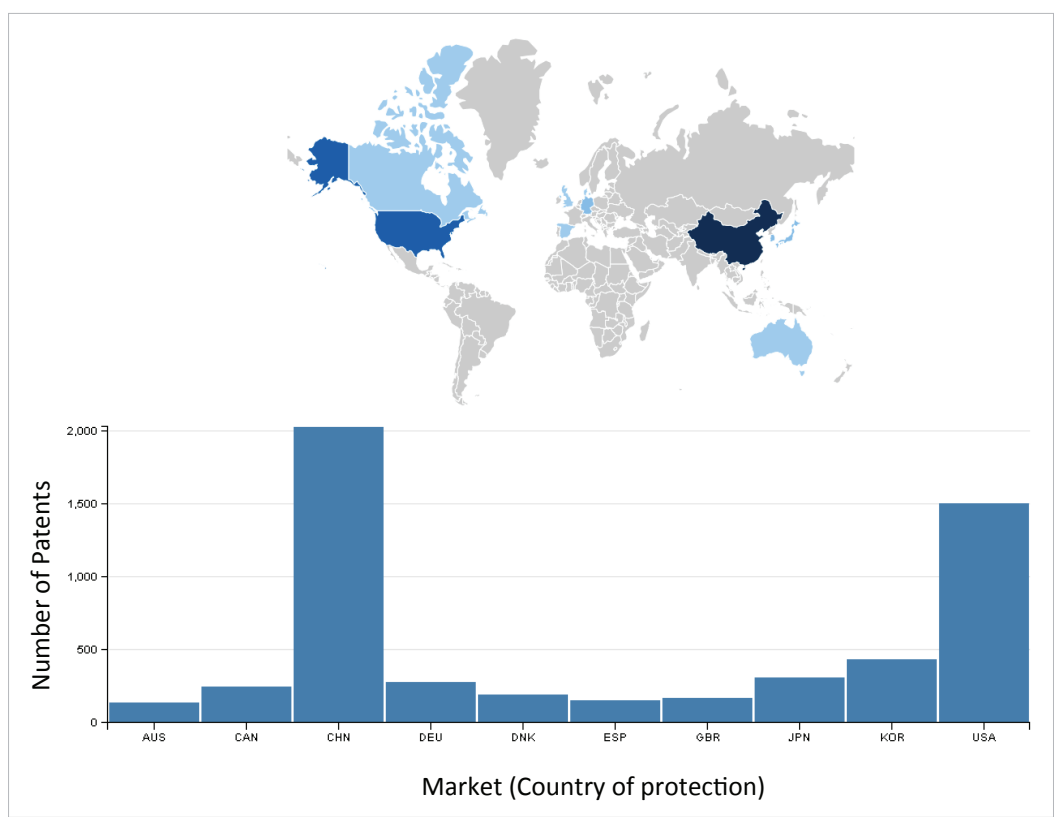

Figura 2. Top 10 países con patentes de energía eólica (fuente: Espacenet)

Tabla 1. Cuota de mercado de productores de energía eólica (fuente: Cleantech, 2012)

\begin{tabular}{|c|l|c|c|}
\hline \multirow{2}{*}{ Ranking } & \multirow{2}{*}{ Nombre empresa } & \multicolumn{2}{|c|}{ Cuota de mercado } \\
\cline { 3 - 4 } & & $\mathbf{2 0 1 1}$ & $\mathbf{2 0 1 0}$ \\
\hline 1 & Vestas & 12,7 & 14,8 \\
\hline 2 & Sinovel & 9,0 & 11,1 \\
\hline 3 & Goldwind & 8,7 & 9,5 \\
\hline 4 & Gamesa & 8,0 & 6,6 \\
\hline 5 & Enercon & 7,8 & 7,2 \\
\hline 6 & General Electric & 7,7 & 9,6 \\
\hline 7 & Suzlon & 7,6 & 6,9 \\
\hline 8 & Guodian United Power & 7,4 & 4,2 \\
\hline 9 & Siemens & 6,3 & 5,9 \\
\hline 10 & MingYang Wind Power & 3,6 & - \\
\hline
\end{tabular}

3. Análisis. Esta etapa es crítica, y consiste en interpretar los datos desde un punto de vista estratégico (inteligencia) determinando su relevancia para la toma de decisiones. Para el análisis se usan varios tipos de indicadores y técnicas (indicadores de patentes, análisis semántico, análisis DAFO, benchmarking, las cinco fuerzas de Porter, etc.). Es conveniente que el sistema de inteligencia sea colaborativo pues ello potencia la creación de nuevas ideas.

4. Difusión. Los resultados se ponen a disposición de los mandos superiores que deben tomar las decisiones estratégicas.

El tratamiento de grandes cantidades de datos requiere una potencia de procesamiento y un espacio de almacenamiento enorme. Usando centros de proceso externos (en la nube o cloud computing) las compañías han hallado la solución a este problema. Como se sabe, la nube es un modelo de 'servicio bajo demanda' que proporciona recursos informáticos (redes, servidores, almacenamiento, aplicaciones y servicios) con un esfuerzo de gestión mínimo por parte de la empresa usuaria, reduciendo el coste de inversión y de operación. Generalmente se paga una suscripción con una tasa mensual (Mell; Grance, 2011). El uso de la nube hace realidad la idea de "todo como un servicio" (everything as a service, XaaS) -es decir, las organizaciones clientes se suscriben a un servicio y no tienen que comprar ni hardware ni software para procesar sus datos, ni realizar ningún mantenimiento.

E-intelligent, una empresa spin-off de la UPM que ofrece productos y servicios de vigilancia tecnológica e inteligencia competitiva, tiene el producto Vicubocloud como plataforma de inteligencia en la nube para satisfacer las necesidades informativas de sus clientes. En este artículo se presentan tres ejemplos recientes.

\section{Metodología}

El proceso de vigilancia tecnológica e inteligencia competitiva (VT\&IC) que presenta este estudio se estructura, tal como recomiendan las normativas europeas en I+D (UNE 166006:2011 y Comité AEN/CTN 166, 2011), en las cuatro etapas descritas anteriormente. VicuboCloud se usa para analizar y mostrar la información relevante en gráficos, lo que permite evaluar de forma sencilla la posición de la compañía en el mercado frente a sus competidores.

\section{Caso 1: Sector de energías renovables. Quién es quién en la fabricación de turbinas eólicas}

Los fabricantes de turbinas eólicas las diseñan, prueban, fabrican y luego prestan asistencia para su operación y man- 
tenimiento. Se trata de un mercado global, cambiante, del que es necesario seguir los movimientos para estar al día de alertas y oportunidades. Se analizó quién es quién entre los suministradores de tecnología eólica y dónde están vendiendo, para lo cual se utilizaron informes de mercado y de patentes, ambos incluidos en las bases de datos de VicuboCloud. Los líderes tecnológicos se pueden identificar no sólo por su volumen de producción, sino también por sus patentes.

La figura 1 muestra los 10 líderes en patentes de turbinas eólicas en los últimos tres años. Vestas, General Electric, Mitsubishi Heavy, Siemens y Wobben Alloys son los principales solicitantes de patentes que aportan desarrollos tecnológicos al mercado. El caso de Wobben es digno de tenerse en cuenta ya que es el inventor y no el solicitante el que aparece en la patente, lo cual puede ser parte de una estrategia en la gestión de patentes más amplia por parte la compañía.

Las patentes pueden cruzarse con los fabricantes de turbinas obtenidos a partir de informes sectoriales actualizados. En 2011, un año difícil y de resultados financieros decepcionantes, la danesa Vestas, líder en tecnología, mantuvo su posición también como líder en la fabricación de turbinas, por encima de Sinovel (sin embargo, la situación cambió en 2012, ya que Vestas perdió su posición frente a General Electric).

En cuanto a dónde están vendiendo los suministradores de turbinas eólicas, según el último informe de mercado de Global Wind Energy Council (GWEC) (2013), China es el mayor mercado de turbinas, tanto tecnológico como mercantil, con tendencia de

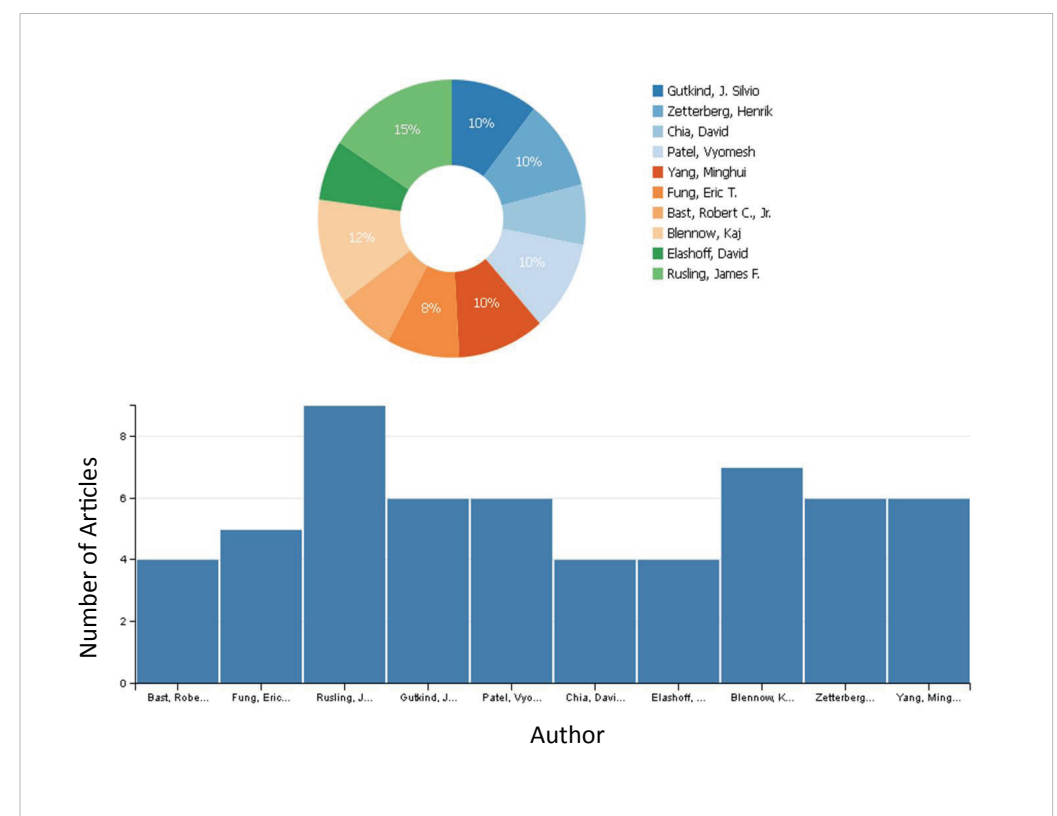

Figura 3. Top 10 autores para el desarrollo de biomarcadores (fuente: Web of knowledge)

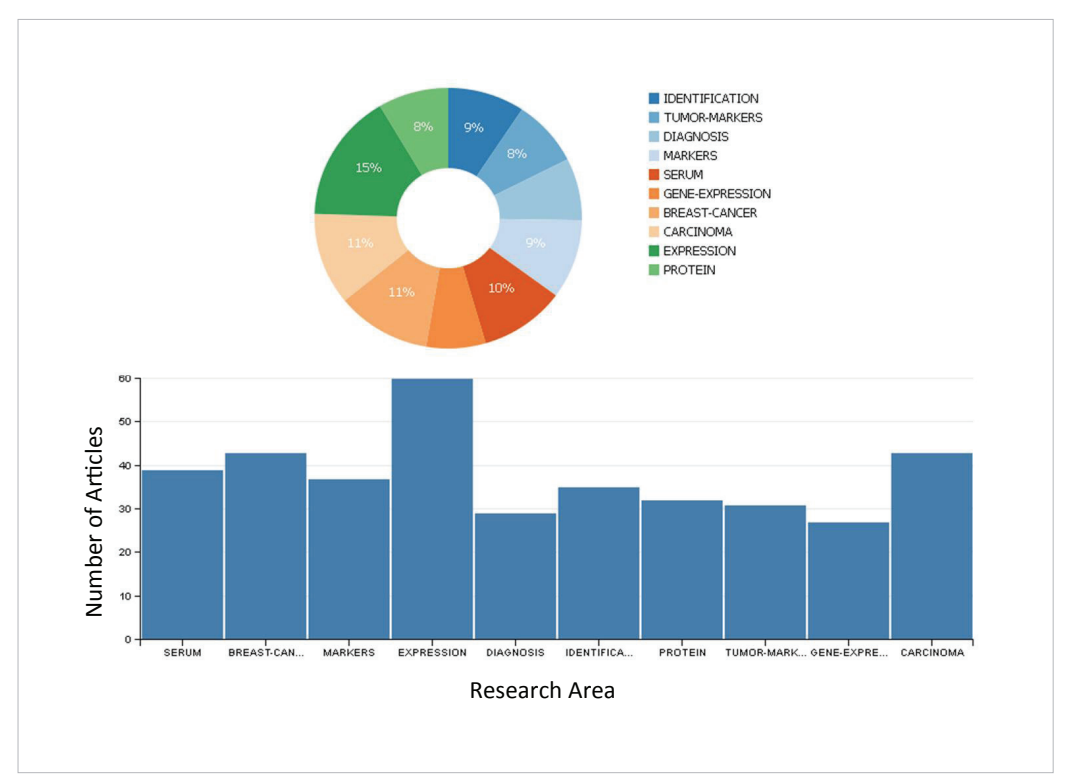

Figura 4. Top 10 áreas de investigación para el desarrollo de biomarcaodores (fuente: Espacenet)

Tabla 2. Comparativa del proceso de calificación de biomarcadores por parte de EMA y FDA

\begin{tabular}{|l|l|l|}
\hline \multirow{2}{*}{ Objetivo } & \multicolumn{1}{|c|}{ Objetivos y resultados formalmente calificados } \\
\cline { 2 - 3 } & \multicolumn{1}{|c|}{ European Medicines Agency } & \multicolumn{1}{c|}{ Food and Drug Administration } \\
\hline Enfoque & $\begin{array}{l}\text { Abordar métodos de diseño y elaboración de fárma- } \\
\text { cos innovadores y específicos }\end{array}$ & $\begin{array}{l}\text { Identificar nuevas herramientas que permitan una produc- } \\
\text { ción más rápida de los medicamentos para que así lleguen } \\
\text { antes a los pacientes }\end{array}$ \\
\hline Calificación formal & $\begin{array}{l}\text { Asesoramiento y colaboración entre el CHMPa y las } \\
\text { empresas, individuales o consorcios }\end{array}$ & $\begin{array}{l}\text { Asesoramiento y colaboración entre la FDA y las empresas, } \\
\text { individuales o consorcios }\end{array}$ \\
\hline Después de la calificación & $\begin{array}{l}\text { Opinión de calificación del CHMP: aceptabilidad de } \\
\text { un método definido para un propósito específico } \\
\text { para el desarrollo de fármacos. } \\
\text { Consejos de calificación del CHMP: orientación sobre } \\
\text { futuros estudios }\end{array}$ & $\begin{array}{l}\text { Declaración para la calificación: Adecuación de los datos pre- } \\
\text { sentados para obtener la calificación y el contexto de uso de } \\
\text { la herramienta de desarrollo de fármacos (drug development } \\
\text { tool, DDT) (abierta al público) }\end{array}$ \\
\hline Biomarcadores calificados & $\begin{array}{l}\text { E biomarcadores: } 2 \text { de nefrotoxicidad y } 4 \text { de Alzhei- } \\
\text { mer }\end{array}$ & $\begin{array}{l}\text { 3 biomarcadores: } 2 \text { de nefrotoxicidad y } 1 \text { de daño morfoló- } \\
\text { gico cardíaco }\end{array}$ \\
\hline
\end{tabular}

a. Committee for Medicinal Products for Human Use 


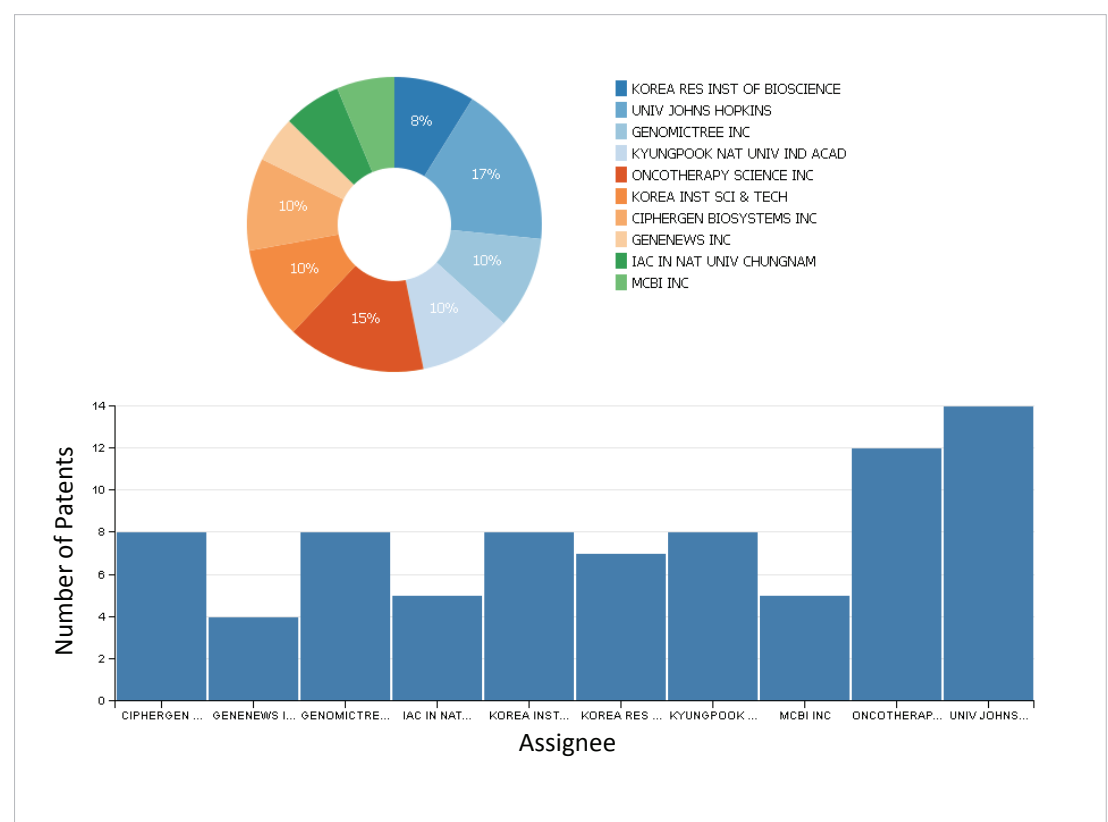

Figura 5. Top 10 líderes de patentes en biomarcadores (fuente: Espacenet)

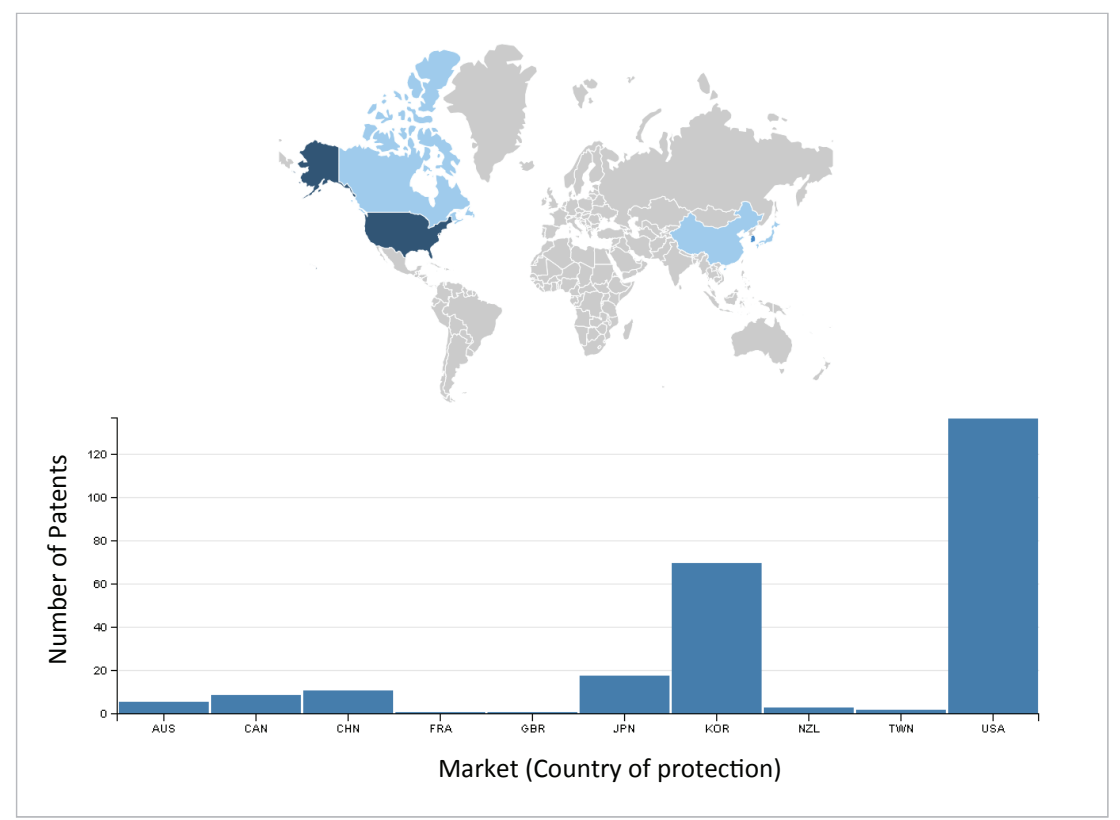

Caso 2: Sector biotecnológico. Estado de la técnica en biomarcadores y barreras a su comercialización

Un biomarcador es una característica que es medida y evaluada como indicador de procesos biológicos normales, procesos patogénicos, o respuestas farmacológicas a una intervención terapéutica. En medicina, un biomarcador puede ser una sustancia detectable que se introduce en un organismo para examinar la función de un órgano o de otros aspectos de la salud; una sustancia cuya presencia anómala indica un estado de enfermedad; o un cambio en la expresión o el estado de una proteína que se correlaciona con el riesgo o progresión de una enfermedad, o con la susceptibilidad de la enfermedad a un tratamiento dado.

Como ocurre con los fármacos, antes de poder comercializar esas sustancias o esos procedimientos de detección, deben pasar el control de las correspondientes agencias oficiales. La evaluación o calificación de un biomarcador se define como el proceso probatorio, gradual y adaptado, que relaciona un biomarcador con factores biológicos y clínicos.

El mundo de los biomarcadores es complejo y en constante evolución. En los últimos años tienen un papel cada vez más importante en la industria farmacológica y en el diagnóstico clínico.

La introducción de un biomarcador en el mercado requiere tres fases:

Figura 6. Top 10 países patentadores de biomarcadores (fuente: Espacenet)

crecimiento en mercados extranjeros. China, seguida de Estados Unidos, es el país con más patentes de energía eólica (figura 2).

Toda la información mostrada es parte de un informe más amplio propiedad de E-intelligent.
- descubrimiento: identificación del biomarcador;

- desarrollo del prototipo: se crean ensayos inmunológicos;

- comercialización: los ensayos están listos para su aplicación clínica.

Para nuestro estudio se analizará el proceso de calificación de biomarcadores en los mercados de Europa y EUA, el es-

Tabla 3. Posibles socios para la calificación de biomarcadores (fuente: VicuboCloud ${ }^{\mathrm{TM}}$ )

\begin{tabular}{|c|c|c|c|c|}
\hline & \multicolumn{4}{|c|}{ Partners information } \\
\hline & Scientific area & Contact & Source & Date \\
\hline 1 & Bladder cancer progression & Aarhus University Hospital, Denmark & $\begin{array}{l}\text { Result from the EU funded FP7- } \\
\text { Health programme }\end{array}$ & 2013-01-07 \\
\hline 2 & Cancer diagnosis, prognosis and follow & Spanish National Research Council & Technology market Europe & 2013-02-11 \\
\hline 4 & Respiratory chain dysfunction & Finnish research group & Technology market Europe & 2013-01-15 \\
\hline 8 & Early diagnosis Alzheimer disease & $\begin{array}{l}\text { Four Spanish public organisations and } \\
\text { the University of Singapore }\end{array}$ & Technology market Europe & $2013-02-22$ \\
\hline 12 & Breast cancer & French Academic Research Laboratory & Technology market Europe & 2013-02-25 \\
\hline
\end{tabular}


tado de la técnica y las barreras comerciales existentes.

El estado de la técnica se obtiene a partir de la bibliografía científica (para detectar nuevo conocimiento) y de patentes (tendencias tecnológicas y de negocio), y las barreras comerciales se identifican analizando la información del proceso de calificación por parte de las agencias europea (EMA, European Medicines Agency) y norteamericana (FDA, Food and Drug Administration).

\section{Estado de la técnica}

Se monitorizaron los artículos publicados por los expertos (figura 3) y se clasificaron según las áreas en las que se están investigando nuevos biomarcadores (figura 4), lo cual permitió establecer el patrón de evolución de la investigación básica. También se buscaron las patentes solicitadas por las empresas e instituciones del sector, cuyo análisis detectó las tendencias actuales en el negocio de los biomarcadores: líderes, mercados y áreas de trabajo tecnológicas. El líder en tecnología de biomarcadores por número de patentes es la universidad norteamericana Johns Hopkins, seguida por la compañía japonesa Oncotherapy, tal como muestra la figura 5.

La figura 6 muestra los mercados más importantes para la venta de biomarcadores, según los países solicitantes de patentes: USA, Corea y Japón.

Finalmente, se analizaron las áreas tecnológicas de mayor interés en el desarrollo de biomarcadores, y su utilización por parte de los líderes del sector (figura 7).

\section{Barreras comerciales}

La comercialización es una tarea costosa pues el biomarcador y su ensayo deben superar la evaluación o calificación de las agencias farmacológicas, y luego hay que preparar su marketing. El procedimiento del ensayo debe seguir normativas establecidas por los organismos reguladores correspondientes.
En la tabla 2 se compara el proceso de calificación seguido por las agencias europea (EMA, European Medicines Agency) y norteamericana (FDA, Food and Drug Administration).

La solicitud de calificación de un biomarcador es mejor hacerla desde un consorcio o agrupación de instituciones de investigación, pues así se comparten conocimientos, recursos, tecnología, muestras, etc., y se ahorran costes y tiempo. La tabla 3 presenta posibles socios para los proyectos de biomarcadores en diferentes áreas de investigación.

\section{Caso 3: Sector residuos. Análisis competitivo en biofuel}

Los biocombustibles son productos -fuentes de energíagenerados a partir de biomasa o material 'recientemente' vivo. Pueden ser sólidos (madera, cosechas y sus residuos, desechos urbanos, etc.), líquidos (aceite vegetal, aceite residual alimentario, biodiesel, etanol u otros alcoholes, e hidrocarburos biológicos) o gaseoso (biometano, biogas, gases de vertederos o hidrógeno obtenido de biomasa).

Tabla 4. Análisis de posicionamiento competitivo de Fulcrum Bioenergy (fuente: Marketline)

\begin{tabular}{|l|l|l|}
\hline \multirow{2}{*}{$\begin{array}{c}\text { Características competi- } \\
\text { tivas }\end{array}$} & \multicolumn{1}{c|}{ Hecho 1 Hechos importantes } \\
\cline { 2 - 3 } & \multicolumn{1}{|c|}{ 1 } & \multicolumn{1}{c|}{ Hecho 2 } \\
\hline Ventajas competitivas & Economía de las materias primas & Proceso propio a escala comercial \\
\hline Pecnología & Adquirida una licencia para el sistema de gasificación & $\begin{array}{l}\text { Patente para integrar los residuos sólidos municipales en } \\
\text { el proceso del etanol }\end{array}$ \\
\hline Coste de producción & Principalmente etanol & Otros: metanol y propanol \\
\hline Acuerdos de ventas & Empresa grande $\$ 1,30$ por galón & $\begin{array}{l}\text { El proceso de producción genera electricidad para } \\
\text { empresas }\end{array}$ \\
\hline $\begin{array}{l}\text { Operaciones financieras } \\
\text { recientes (2011-2012) }\end{array}$ & $\begin{array}{l}\text { 23-12-2011: Atrae una inversión privada de \$131,21M en } \\
\text { sus instalaciones }\end{array}$ & Acuerdo de ventas de 3 años \\
\hline Inversores & Un grupo renovable & Firma de capital de riesgo empresa invierte en ella \\
\hline Socios y alianzas & Dos socios en materias primas y dos en técnica & Un socio vende \\
\hline Futuros hitos & Construcción de 2 instalaciones adicionales en los EUA & \\
\hline
\end{tabular}


Tabla 5. Análisis DAFO de Fulcrum Bioenergy

\begin{tabular}{|c|c|}
\hline Debilidades & $\begin{array}{l}\text { - Sin fuerza ante posibles competidores (las paten- } \\
\text { tes aún están pendientes) } \\
\text { - Concentración geográfica } \\
\text { - Los costos del producto sólo son estimados; } \\
\text { dependen de múltiples variables }\end{array}$ \\
\hline Amenazas & $\begin{array}{l}\text { - Patente sconcedidas a otros } \\
\text { - Leyes y reglamentos ambientales } \\
\text { - Aumento de la competencia } \\
\text { - Cambios en los precios de los bienes de consumo }\end{array}$ \\
\hline Fortalezas & $\begin{array}{l}\text { - Contratos rentables para obtener materia prima } \\
\text { - Proceso propietario a escala comercial } \\
\text { - No hay nuevas redes de logística para el transpor- } \\
\text { te de la materia prima } \\
\text { - Promotor / propietario / operador }\end{array}$ \\
\hline Oportunidades & $\begin{array}{l}\text { - Instalaciones nuevas } \\
\text { - Aumento de la demanda de servicios ambientales } \\
\text { - Nuevas iniciativas de "energía de los residuos" } \\
\text { - Extensión de la patente a nuevos mercados }\end{array}$ \\
\hline
\end{tabular}

\section{Informe}

Una compañía en el sector del biocombustible quiere analizar la información disponible de diferentes competidores tecnológicos para implementar una estrategia de posicionamiento competitivo en el área de los residuos sólidos urbanos para la producción de biomasa.

Primeramente se analiza la compañía para identificar sus modelos de negocio, acuerdos con otras compañías, desarrollos tecnológicos y noticias financieras. Está situada en California y diseña, desarrolla, posee y gestiona instalaciones que convierten residuos de celulosa en etanol. Se presenta el análisis competitivo de posicionamiento del competidor dentro del sector (tabla 4).

La evaluación final de las ventajas y debilidades competitivas se muestra en el análisis DAFO de la tabla 5, que apoya la toma de decisiones sobre la estrategia a seguir.

\section{Conclusiones}

El número de factores que influyen en la estrategia de negocio ha aumentado notablemente debido al fenómeno de la globalización y a la situación económica en Europa y EUA. La apertura de nuevos mercados en todo el mundo no es sólo tarea de grandes empresas, sino de todas, independientemente de su tamaño, con el fin de sobrevivir en este contexto económico sumamente competitivo.

Como el entorno en el que se mueven las compañías se hace cada vez más turbulento, las decisiones de los directivos se complican: deben buscar nuevas oportunidades de negocio en todos los mercados a la vez que lidiar con nuevos competidores en su propio entorno.

\section{Anexo: Características principales de VicuboCloud}

VicuboCloud es una plataforma que permite a una organización realizar vigilancia tecnológica e inteligencia competitiva de la Web y extraer la información estratégica necesaria. Se caracteriza por ser un servicio en "la nube". Por ello no es necesario ningún tipo de instalación en servidores propios ni licencias de software, con lo que se evitan los costes y molestias asociados al mantenimiento de equipos informáticos. https://www.vicubocloud.es
Sus funciones principales son:

- monitorizar cualquier fuente de información;

- centralizar y sistematizar el proceso de vigilancia tecnológica e -inteligencia competitiva (VT\&IC);

- reducir el tiempo dedicado a la gestión de información;

- atención personalizada y confidencial;

- facilitar la integración de información de forma segura;

- analizar visualmente la información clasificada;

VicuboCloud incorpora tres extractores de información:

- Vicubo sectores: Información de partida clasificada por sectores, alimentada por fuentes de información abierta y contrastadas por un equipo de analistas. Además ofrece licitaciones, ayudas, legislación, normas, proyectos de I+D, informes económicos, análisis de mercado, estadísticas, patentes, publicaciones, artículos, eventos, noticias, etc.

- Vicubo social: información de las redes sociales configurable, capaz de rastrear, medir y evaluar en tiempo real la información generada en Facebook, Twitter, LinkedIn, Google+, etc.

- Vicubo reader: permite recuperar automáticamente las novedades producidas en las fuentes monitorizadas, lo cual elimina la necesidad de acudir a ellas diariamente.

Cada organización puede contratar lo que le interese y durante el tiempo que desee. Se puede alquilar por períodos de tiempo determinados, según la duración del informe. Permite integrar fuentes de información de pago (Thomson Reuter, Factiva, Lexis Nexis, etc.). Su potencia radica en el análisis de información mediante gráficos y la posibilidad de compartir información y recibir alertas.

\section{Bibliografía}

Comité Aenor AEN/CTN 166 (2011). UNE 166.006:2011. Gestión de la I+D+i: Sistema de vigilancia tecnológica e inteligencia competitiva.

http://www.aenor.es/aenor/normas/normas/fichanorma. asp ?tipo $=N \&$ codigo $=N 0046930$

Fuld \& Company (2002). Measuring the value of competitive intelligence: the inadecuacies of return-on-investment calculation. White paper, Fuld \& Company, Cambridge, MA. http://www.fuld.com/wp/measuring-the-value-ofcompetitive-intelligence

Fuld, Leonard M. (1985). Competitor intelligence: how to get it; how to use it. New York: John Wiley \& Sons. ISBN: 978 0471809678

Herring, Jan P. (1999). "Key intelligence topics: a process to identify and define intelligence needs". Competitive intelligence review, v. 10, n. 2, pp. 4-14.

ht t p://dx.doi.org/10.1002/(S/CI) 1520 6386(199932)10:2<4::AID-CIR3>3.3.CO;2-3

McKinnon, Sharon; Burns, William (1992). The information mosaic. Boston: Harvard Business School Press. ISBN: 978 0875843179

Mell, Peter; Grance, Timothy (2011). The NIST definition of cloud computing. NIST special publication 800-145. http://csrc.nist.gov/publications/nistpubs/800-145/SP800-145.pdf 